\title{
Selection Criteria for Active Surveillance of Patients with Prostate Cancer in Korea: A Multicenter Analysis of Pathology after Radical Prostatectomy
}

\author{
Chang Wook Jeong, MD, PhD ${ }^{1}$ \\ Sung Kyu Hong, MD, PhD² \\ Seok Soo Byun, MD, PhD² \\ Seong Soo Jeon, $\mathrm{MD}, \mathrm{PhD}^{3}$ \\ Seong II Seo, MD, $\mathrm{PhD}^{3}$ \\ Hyun Moo Lee, MD, PhD 3 \\ Hanjong Ahn, MD, PhD ${ }^{4}$ \\ Dong Deuk Kwon, MD, $\mathrm{PhD}^{5}$ \\ Hong Koo Ha, MD, PhD ${ }^{6}$ \\ Tae Gyun Kwon, MD, $\mathrm{PhD}^{7}$ \\ Jae Seung Chung, MD, $P h D^{8}$ \\ Cheol Kwak, MD, PhD ${ }^{1}$ \\ Hyung Jin Kim, MD, PhD ${ }^{9}$
}

${ }^{*}$ A list author's affiliations appears at the end of the paper.

\begin{abstract}
Purpose
Korean patients with prostate cancer (PC) typically present with a more aggressive disease than patients in Western populations. Consequently, it is unclear if the current criteria for active surveillance (AS) can safely be applied to Korean patients. Therefore, this study was conducted to define appropriate selection criteria for AS for patients with PC in Korea.
\end{abstract}

\section{Materials and Methods}

We conducted a multicenter retrospective study of 2,126 patients with low risk PC who actually underwent radical prostatectomy. The primary outcome was an unfavorable disease, which was defined by non-organ confined disease or an upgrading of the Gleason score to $\geq 7$ (4+3). Predictive variables of an unfavorable outcome were identified by multivariate analysis using randomly selected training samples ( $n=1,623,76.3 \%)$. We compared our selected criteria to various Western criteria for the primary outcome and validated our criteria using the remaining validation sample $(n=503,23.7 \%)$.

\section{Results}

A non-organ confined disease rate of $14.9 \%$ was identified, with an increase in Gleason score $\geq 7(4+3)$ of $8.7 \%$ and a final unfavorable disease status of $20.8 \%$. The following criteria were selected: Gleason score $\leq 6$, clinical stage T1-T2a, prostate-specific antigen (PSA) $\leq 10 \mathrm{ng} / \mathrm{mL}$, PSA density $<0.15 \mathrm{ng} / \mathrm{mL} / \mathrm{mL}$, number of positive cores $\leq 2$, and maximum cancer involvement in any one core $\leq 20 \%$. These criteria provided the lowest unfavorable disease rate (11.7\%) when compared to Western criteria (13.3\%-20.7\%), and their validity was confirmed using the validation sample (5.9\%).

\section{Conclusion}

We developed AS criteria which are appropriate for Korean patients with PC. Prospective studies using these criteria are now warranted.

Key words

Prostatic neoplasms, Observation, Prostatectomy, Pathology
Correspondence: Cheol Kwak, MD, PhD Department of Urology, Seoul National University Hospital, Seoul National University College of Medicine, 101 Daehak-ro, Jongno-gu, Seoul 03080, Korea

Tel: 82-2-2072-7628

Fax: 82-2-742-4665

E-mail:mdrafael@snu.ac.kr

Received October 5, 2016

Accepted April 6, 2017

Published Online April 14, 2017 


\section{Introduction}

Active surveillance (AS) is a treatment option, with curative intent, that is widely accepted in many cancer treatment guidelines for patients with very low risk prostate cancer (PC) $[1,2]$. Currently, there are insufficient long-term data regarding the outcomes of patients with $\mathrm{PC}$ to reliably identify individuals who would be suitable for AS, as well as to define standardized criteria for definitive treatment of patients who are on AS. Indeed, only one study is currently available, which consisted of a 15-year follow-up of AS in patients with PC [3]. In their study, Klotz et al. [3] reported an overall survival (OS) rate of $62 \%$ and a disease-specific survival (DSS) of $94.3 \%$, with $27 \%$ of patients undergoing definitive treatment over the 15-year period. At the end of the 15 -year observation period, $55.0 \%$ of patients remained untreated and on surveillance. Therefore, a significant proportion of patients initially placed on AS required definitive treatment, and some of these did not receive treatment at the appropriate time. Therefore, the major challenge of AS is to differentiate patients with significant PC disease status from those with an insignificant disease status.

The heterogeneous nature of PC is the principal factor underlying our current inability to specifically identify those patients in whom the disease is likely to progress [4]. Identification of standardized criteria for definitive treatment is further complicated by ethnicity-specific differences in the disease course of PC. As an example, in Asian populations, including Koreans, patients with PC tend to have a higher Gleason score and advanced stage than their Western counterparts [5-7]. Thus, the current AS selection criteria, which are based on Western studies, should cautiously be applied to Asian populations [8,9], which limits the widespread use of AS in Asia. Therefore, the development of more stringent AS protocols for Asian patients based on well-designed, large clinical studies, is urgently needed [10]. Accordingly, this multicenter retrospective study was conducted to identify potentially appropriate selection criteria for AS in Korean patients with PC. A secondary goal was to compare the validity of our new criteria to various Western criteria for our study population.

\section{Materials and Methods}

\section{Ethic statement}

This study was approved by the Institutional Review Board (IRB) of Seoul National University Hospital (H-1507-
005-683) and each participating institution.

\section{Study design and patient eligibility}

We conducted a multicenter, retrospective study among Korean patients with PC who would now be potential candidates for AS, but actually underwent radical prostatectomy between 2000 and 2012. Eight institutions were included in the study, Seoul National University Hospital, Seoul National University Bundang Hospital, Samsung Medical Center, Asan Medical Center, Pusan National University Hospital, Chonnam National University Hwasun Hospital, Kyungpook National University Hospital, and Inje University Haeundae Paik Hospital. As these were low-risk patients, only pelvic lymph node dissection was performed for selected cases (about 20\%). We evaluated pathological outcomes and biochemical recurrence (BCR)-free survival. Inclusion criteria for patient selection were as follows: histologically proven adenocarcinoma of the prostate, diagnosed using contemporary transrectal ultrasound-guided prostate biopsy ( $\geq 10$ cores), with a clinical staging of T1c-T2a; prebiopsy prostate-specific antigen (PSA) $\leq 10 \mathrm{ng} / \mathrm{mL}$; and a biopsy Gleason score $\leq 6$. The exclusion criteria were as follows: prior radiation therapy; androgen deprivation therapy or any other treatment for PC, such as high-intensity focused ultrasound; previous prostate surgery, including transurethral resection of the prostate; or missing data pertaining to essential variables.

\section{Outcomes}

The following key pathological features were evaluated from the radical prostatectomy pathology report: non-organ confined disease, any upgrading of the Gleason score, a specific increase in the Gleason score $\geq 7(4+3)$, an unfavorable disease outcome, and insignificant cancer. The primary outcome for analysis was an unfavorable disease status, defined as non-organ confined disease or an upgrading of the Gleason score $\geq 7(4+3)$. Insignificant cancer was defined as follows according to the Stamey criteria: organ-confined disease; a Gleason score $\leq 6$; and a tumor volume $<0.5 \mathrm{~mL}$ [11]. BCRfree survival, DSS and OS were also evaluated.

\section{Statistics}

Patients were randomly assigned to two groups, with about $75 \%$ of patients allocated to the 'training sample' used to identify the best selection criteria, and the remaining $25 \%$ assigned to the 'validation sample' used to externally validate the selection criteria. Random allocation was performed using SPSS ver. 21.0 (IBM Corp., Armonk, NY) as previously described [12]. Finally, 1,623 (76.3\%) were included to the 
Table 1. Basic characteristics of the patients $(n=2,126)$

\begin{tabular}{|c|c|c|}
\hline Characteristic & Training sample $(\mathrm{n}=1,623)$ & Validation sample $(\mathrm{n}=503)$ \\
\hline Age (yr) & $64.7 \pm 6.9(37-86)$ & $65.0 \pm 7.2(39-81)$ \\
\hline Prostate size $(\mathrm{mL})$ & $39.0 \pm 17.4(7.8-141.0)$ & $39.0 \pm 17.8(12.0-162.0)$ \\
\hline PSA (ng/mL) & $5.4 \pm 2.0(0.1-10.0)$ & $5.3 \pm 1.9(0.7-9.9)$ \\
\hline PSA density (ng/mL/mL) & $0.16 \pm 0.09(0-0.60)$ & $0.15 \pm 0.08(0.02-0.51)$ \\
\hline \multicolumn{3}{|l|}{ Clinical T stage } \\
\hline $\mathrm{T} 1 \mathrm{c}$ & $1,007(62.0)$ & $334(66.4)$ \\
\hline $\mathrm{T} 2 \mathrm{a}$ & $616(38.0)$ & $169(33.6)$ \\
\hline \multicolumn{3}{|l|}{ No. of positive biopsy cores } \\
\hline 1 & $750(46.2)$ & $227(45.1)$ \\
\hline 2 & $350(21.6)$ & $126(25.0)$ \\
\hline$>2$ & $523(32.2)$ & $150(29.8)$ \\
\hline Maximum involvement of core $(\%)$ & $25.5 \pm 21.0(0.3-100)$ & $25.1 \pm 20.5(0.5-100)$ \\
\hline \multicolumn{3}{|l|}{ Operation methods } \\
\hline Open & 767 (47.3) & $238(47.3)$ \\
\hline Laparoscopic & $178(11.0)$ & $65(12.9)$ \\
\hline Robotic & $678(41.8)$ & $200(39.8)$ \\
\hline \multicolumn{3}{|l|}{ Pathologic T stage } \\
\hline T0 & $8(0.5)$ & $4(0.8)$ \\
\hline $\mathrm{T} 2 \mathrm{a}-\mathrm{b}$ & $371(22.9)$ & $104(20.7)$ \\
\hline $\mathrm{T} 2 \mathrm{c}$ & $1,008(62.1)$ & $315(62.6)$ \\
\hline T3a & $214(13.2)$ & $74(14.7)$ \\
\hline $\mathrm{T} 3 \mathrm{~b}$ & $19(1.2)$ & $6(1.2)$ \\
\hline $\mathrm{T} 4$ & $3(0.2)$ & 0 \\
\hline Lymph node involvement & $4(0.2)$ & $1(0.2)$ \\
\hline \multicolumn{3}{|l|}{ Pathologic Gleason score } \\
\hline$\leq 6$ & $810(49.9)$ & $253(50.3)$ \\
\hline $7(3+4)$ & $666(41.0)$ & $211(41.9)$ \\
\hline $7(4+3)$ & $115(7.1)$ & $31(6.2)$ \\
\hline 8 & $20(1.2)$ & $4(0.8)$ \\
\hline 9 & $12(0.7)$ & $4(0.8)$ \\
\hline Positive surgical margin & $281(17.3)$ & $75(14.9)$ \\
\hline \multicolumn{3}{|l|}{ Follow-up duration (mo) } \\
\hline Median & 53 & 51 \\
\hline Mean \pm SD & $55.2 \pm 28.0$ & $54.1 \pm 27.2$ \\
\hline
\end{tabular}

Values are presented as mean \pm SD (range) or number (\%). PSA, prostate specific antigen; SD, standard deviation.

training sample and $503(23.7 \%)$ in the validation sample.

Using the training sample, the association between preoperative clinicopathological factors from various AS criteria [3,13-17] with primary and major secondary outcomes was evaluated. The following factors were evaluated: age; clinical stage (T1c, T2a); prostate size; PSA; PSA density, measured both as a continuous variable and categorical variable $(<0.15$, $0.15-0.2$, and $>0.2 \mathrm{ng} / \mathrm{mL} / \mathrm{mL})$; number of positive cores (1, 2 , and $\geq 3$ ); percent positive cores, defined as the number of positive cores/number of total cores; the maximum cancer involvement rate in any core, measured both as a continuous and categorical variable $(\leq 20 \%,>20 \%$ and $\leq 50 \%$, and $>50 \%)$. Univariate logistic regression analyses were conducted to assess the association of each factor with an unfavorable disease outcome.

Subsequently, multivariate analysis with backward elimination using a probability for removal of 0.1 was performed. This analysis included all variables found to be significant by univariate analyses as a starting point. If both continuous and categorical forms of the same variable were significant upon univariate analysis, we selected a categorical form for better interpretation on multivariate analysis. The purpose 
of the multivariate analysis was to identify significant factors that would be used as selection criteria. For the analysis, we employed backward elimination, which can find the best fitted, reduced model. To rule out the effects of heterogeneity of the institutions, we conducted sensitivity analysis. We compared two multivariate logistic regression models, an original one and another model including institutions as the variables.

Univariate and multivariate Cox proportional regression analyses were performed to assess BCR-free survival using the same method. We also used a spline curve fitting method to visualize the odds ratio (OR) and hazard ratio (HR) according to significant continuous variables and to identify sensitive cut-off values for each retained significant factor. The results of these analyses were then used to develop new AS selection criteria for Korean patients with PC. Finally, we compared the validity of our selection criteria against various Western criteria $[3,13-17]$ in our study population in terms of key pathological features and BCR-free survival. We subsequently validated our criteria using the validation sample.

For statistical comparisons, a p-value of $<0.05$ was considered significant. Statistical analyses were performed using SPSS ver. 21.0, with curve fitting performed using $\mathrm{R}$ for Windows ver. 3.1.1 (R Foundation for Statistical Computing, Vienna, Austria).

\section{Results}

\section{Patients' characteristics}

Across the eight participating institutions, 2,433 patients met our inclusion criteria during the defined study period. After screening for exclusion criteria, the data of 2,126 patients were retained for the final analysis. Basic characteristics of our study cohort are summarized in Table 1. Overall, non-organ confined disease was identified in $14.9 \%(316 / 2,126)$ of cases, with a general upgrading of the Gleason score in $50.0 \%(1,063 /$ $2,126)$ of cases and an upgrading to a score $\geq 7(4+3)$ in $8.7 \%$ $(186 / 2,126)$ of cases. A final unfavorable disease status was defined in $20.8 \%(442 / 2,126)$ of cases. At the median followup period of 52 months, the 5- and 10-year BCR-free survival rate was $92.3 \%$ and $78.3 \%$, respectively (Fig. 1). The 10-year DSS and OS were $97.7 \%$ and $95.8 \%$, respectively.

\section{Factors associated with unfavorable disease outcome and BCR-free survival}

The results of logistic regression analyses are reported in Table 2. With the exception of clinical stage, all variables eval-

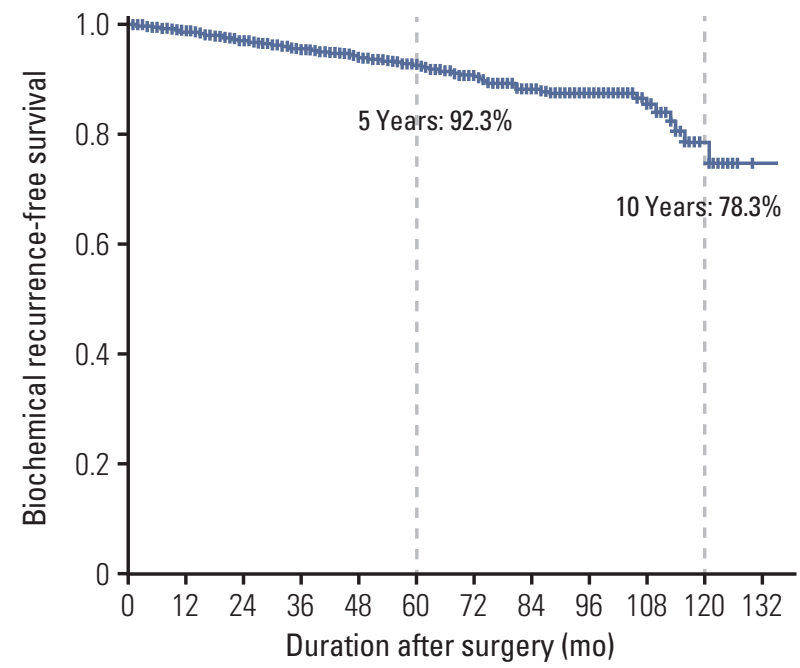

Fig. 1. Biochemical recurrence-free survival curve.

uated by univariate logistic regression analysis were significantly associated with an unfavorable disease outcome. Among these, age, PSA density (as a categorical variable), number of positive biopsy cores and the maximum cancer involvement rate in any core (as a categorical variable) were retained as significant factors upon multivariate analysis. In the sensitivity analysis, the final multivariate logistic regression model was not significantly affected by the effects of the institutions (S1 Table).

Cox proportional regression analyzes are presented in Table 3. BCR-free survival was inversely associated with clinical stage, a PSA density $>0.2 \mathrm{ng} / \mathrm{mL} / \mathrm{mL}$ and the maximum cancer involvement rate in any core (as categorical variable) upon multivariable analysis. The curve fitting analysis is presented in Fig. 2. A PSA density $\geq 0.15 \mathrm{ng} / \mathrm{mL} / \mathrm{mL}$ was associated with a higher risk of unfavorable disease $(\mathrm{OR}>1)$ and a higher risk of $B C R(H R>1)$. From this curve, we identified 1 or 2 positive biopsy cores and a maximum cancer involvement in any core $\leq 20 \%$ as cut-off criteria.

\section{Suggestion of an appropriate AS selection criteria for Korean patients with PC, with comparison to Western AS criteria}

Based on the aforementioned results, we identified the following criteria for appropriate AS in Korean patients with PC: a biopsy Gleason score $\leq 6$; a clinical stage T1-T2a; a PSA $\leq 10$ $\mathrm{ng} / \mathrm{mL}$ and a PSA density $<0.15 \mathrm{ng} / \mathrm{mL} / \mathrm{mL}$; a number of positive cores $\leq 2$; and a maximum cancer involvement rate within any core $\leq 20 \%$. Comparison of these criteria to established Western criteria is summarized in Table 4. Using our newly defined criteria, we could select more patients for AS 
Table 2. Logistic regression analyses for unfavorable disease

\begin{tabular}{|c|c|c|c|c|}
\hline & \multicolumn{2}{|c|}{ Univariate } & \multicolumn{2}{|c|}{ Multivariate } \\
\hline & OR $(95 \% \mathrm{CI})$ & $\overline{p \text {-value }}$ & OR $(95 \% \mathrm{CI})$ & p-value \\
\hline Age (yr) & $1.03(1.01-1.04)$ & 0.008 & $1.03(1.01-1.05)$ & $<0.001$ \\
\hline cT stage, T2a vs. T1c & $1.26(0.983-1.60)$ & 0.068 & - & - \\
\hline Prostate size (mL) & $0.98(0.97-0.99)$ & $<0.001$ & - & - \\
\hline PSA (ng/mL) & 1.09 (1.03-1.16) & 0.006 & - & - \\
\hline PSAD (cont.) & $46.83(12.63-173.72)$ & $<0.001$ & - & - \\
\hline PSAD (ng/mL/mL) & & $<0.001$ & & $<0.001$ \\
\hline$<0.15$ & Reference & & Reference & \\
\hline $0.15-0.2$ & $1.37(0.99-1.89)$ & 0.055 & $1.21(0.86-1.72)$ & 0.274 \\
\hline$>0.2$ & $2.09(1.59-2.75)$ & $<0.001$ & $1.85(1.37-2.51)$ & $<0.001$ \\
\hline Positive biopsy cores & & $<0.001$ & & 0.008 \\
\hline 1 & Reference & & Reference & \\
\hline 2 & $1.33(0.96-1.84)$ & 0.091 & $1.24(0.87-1.76)$ & 0.232 \\
\hline$\geq 3$ & 2.07 (1.58-2.73) & $<0.001$ & $1.62(1.17-2.25)$ & 0.004 \\
\hline Positive cores $(\%)$ & $1.02(1.01-1.03)$ & $<0.001$ & - & - \\
\hline $\begin{array}{l}\text { Maximum cancer involvement } \\
\text { rate in any core (cont.) }\end{array}$ & $1.02(1.01-1.03)$ & $<0.001$ & - & - \\
\hline $\begin{array}{l}\text { Maximum cancer involvement } \\
\text { rate in any core }(\%)\end{array}$ & & $<0.001$ & & $<0.001$ \\
\hline$\leq 20$ & Reference & & Reference & \\
\hline$>20$ and $\leq 50$ & $1.75(1.32-2.31)$ & $<0.001$ & $1.42(1.04-1.92)$ & 0.025 \\
\hline$>50$ & $3.32(2.31-4.77)$ & $<0.001$ & $2.35(1.57-3.50)$ & $<0.001$ \\
\hline
\end{tabular}

OR, odds ratio; $\mathrm{CI}$, confidence interval; cT stage, clinical T stage; PSA, prostate specific antigen; PSAD, PSA density.

( $\mathrm{n}=402$ ) than when using the Johns Hopkins University criteria ( $\mathrm{n}=381$ ), which is considered to be the most stringent criteria for AS selection among training samples (Table 5). Our criteria also provided a lower unfavorable disease rate $(11.7 \%)$ and higher insignificant cancer rate $(12.0 \%)$ in our training sample than the Western criteria. The 10-year BCR-free survival was also the highest $(91.7 \%)$ among all eligibility criteria. Furthermore, the unfavorable disease rate was only $5.9 \%$, with a 10 -year BCR-free survival of $96.7 \%$ in our validation sample, which confirmed our original results.

\section{Discussion}

PC has been shown to be more aggressive in Asian populations than in Western populations due to ethnicity-specific factors and overall lower access to PSA screening [18]. Specifically for radical prostatectomy cases, even after adjusting for other factors, the Gleason score for Korean men is consistently higher and the disease status is more advanced than in African-American men, who are already at a significantly higher risk than Caucasian men for aggressive PC [19]. Moreover, the Gleason score has been reported to be higher for Asian-American patients than for Caucasian men, despite equal access to healthcare [20]. These epidemiological data clearly demonstrate the need for AS criteria specific to Asian populations. A study using the Johns Hopkins University criteria to evaluate a study group of patients who had undergone radical prostatectomy, revealed an upgrading of the Gleason score in $17.1 \%(322 / 1,892)$ of their cases, with an unfavorable disease outcome, defined as a non-organ confined disease or any upgrading of the Gleason score, in 22.3\% $(419 / 1,892)$ of their cases [21]. When applying this criteria and definition to our entire cohort, our rate of any upgrading of the Gleason score was 40.5\% (210/518), with an unfavorable disease rate occurring in $43.1 \%(233 / 518)$ of cases. A recently published multicenter study in Japan reported a pathological outcome similar to ours [22], with a rate of upgrading of the Gleason score of $52.8 \%(150 / 284)$, and $14.1 \%$ (40/284) for upgrading to $\geq 7(4+3)$. This study used the same definition of unfavorable disease outcome as our study, reporting an unfavorable disease rate of $21.1 \%$ 
Table 3. Cox proportional regression analyses for biochemical recurrence-free survival

\begin{tabular}{|c|c|c|c|c|}
\hline & \multicolumn{2}{|c|}{ Univariate } & \multicolumn{2}{|c|}{ Multivariate } \\
\hline & HR (95\% CI) & p-value & HR (95\% CI) & p-value \\
\hline Age $(\mathrm{yr})$ & $1.00(0.97-1.03)$ & 0.968 & - & - \\
\hline cT stage, T2a vs. T1c & $1.55(1.08-2.23)$ & 0.019 & $1.62(1.09-2.40)$ & 0.017 \\
\hline Prostate size $(\mathrm{mL})$ & $1.00(0.99-1.01)$ & 0.729 & - & - \\
\hline PSA (ng/mL) & $1.15(1.05-1.25)$ & 0.003 & - & - \\
\hline PSAD (cont.) & $24.81(3.96-155.49)$ & $<0.001$ & - & - \\
\hline $\operatorname{PSAD}(\mathrm{ng} / \mathrm{mL} / \mathrm{mL})$ & & 0.003 & & 0.067 \\
\hline$<0.15$ & Reference & & Reference & \\
\hline $0.15-0.2$ & $1.13(0.68-1.88)$ & 0.643 & $1.05(0.61-1.81)$ & 0.856 \\
\hline$>0.2$ & $1.96(1.32-2.91)$ & $<0.001$ & $1.64(1.06-2.53)$ & 0.027 \\
\hline Positive biopsy cores & & 0.008 & & - \\
\hline 1 & Reference & & - & \\
\hline 2 & $1.39(0.86-2.24)$ & 0.184 & - & - \\
\hline$\geq 3$ & $1.75(1.16-2.65)$ & 0.008 & - & - \\
\hline Positive cores (\%) & $1.01(1.00-1.02)$ & 0.037 & - & - \\
\hline $\begin{array}{l}\text { Maximum cancer involvement } \\
\text { rate in any core (concentration) }\end{array}$ & $1.02(1.01-1.02)$ & $<0.001$ & - & - \\
\hline $\begin{array}{l}\text { Maximum cancer involvement } \\
\text { rate in any core }(\%)\end{array}$ & & 0.004 & & 0.039 \\
\hline$\leq 20$ & Reference & & Reference & \\
\hline$>20$ and $\leq 50$ & $1.78(1.17-2.73)$ & 0.008 & $1.64(1.06-2.52)$ & 0.025 \\
\hline$>50$ & $2.22(1.29-3.83)$ & 0.004 & $1.80(1.02-3.16)$ & 0.041 \\
\hline
\end{tabular}

$\mathrm{HR}$, hazard ratio; $\mathrm{CI}$, confidence interval; $\mathrm{cT}$ stage, clinical T stage; PSA, prostate specific antigen; PSAD, PSA density.

$(60 / 284)$. In the present study, we reported rates of up-grading of the Gleason score of $50.0 \%$, upgrading to $\geq 7(4+3)$ of $8.7 \%$, and an unfavorable disease rate of $20.8 \%$ for our entire cohort, which were similar to previously reported trends in Asian populations. These results underline the caution that needs to be exerted when adopting a Western AS protocol for Asian populations.

A similar limitation of the usefulness of Western AS protocols for Asian patients with AS was demonstrated in the International Prostate Cancer Research: Active Surveillance (PRIAS) project, which aimed to prospectively evaluate AS with data from 18 countries, including Japan [23]. Notably, there was a high reclassification rate at the time of the first biopsy in the PRIAS-Japan protocol [24] of 33.8\% compared to $24 \%$ for the global cohort $[23,24]$. Based on these outcomes, the PRIAS-Japan research group agreed that a long-term follow-up is needed to determine if the PRIAS protocol could safely be used in Asian patients with PC. According to the data from Japan, a positive core rate was a strong predictor of reclassification [24]. Indeed, the reclassification rate was significantly lowered to $20.1 \%$ by using one positive core as a strict selection criterion. However, using these criteria, about $25 \%$ of patients who would benefit from AS would lose this opportunity.

From this perspective, we would like to emphasize that our new selection criteria for AS is not the strictest criteria, but we do consider it to be the most appropriate for Asian populations. Applying our criteria, we would select more patients for AS than would be selected using the Johns Hopkins University AS criteria (402 vs. 381, respectively), with these criteria being the most stringent [16]. Moreover, our selection criteria provided a lower rate of unfavorable disease outcome than was obtained using the John Hopkins criteria ( $11.7 \%$ vs. $13.4 \%$, respectively), and a higher BCR-free survival rate $(91.74 \%$ vs. $74.5 \%$, respectively). Therefore, based on our criteria, we could offer AS to a greater proportion of patients with PC, without compromising the identification of patients who would benefit from definitive treatment.

Previous studies have evaluated AS selection criteria for Korean men, with some evidence of their usefulness $[9,25,26]$. However, these were single center studies that included a small number of patients or studies that only evaluated the applicability of Western AS criteria to Korean men. One Japanese prospective AS study attempted to develop their own AS protocol [27]. However, their selection of crite- 
A

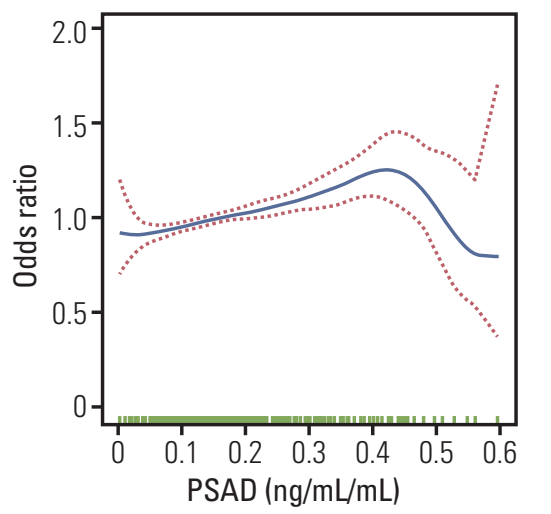

D

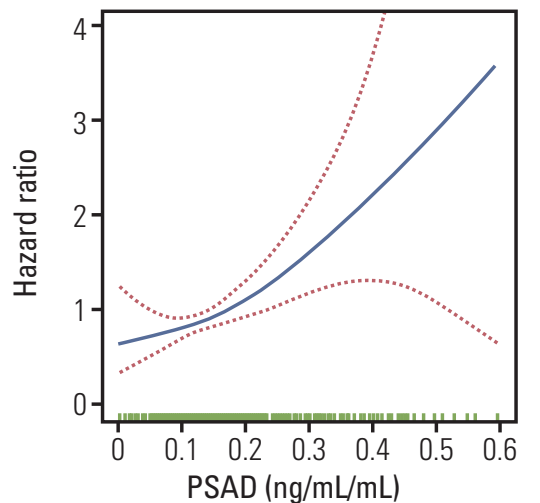

B

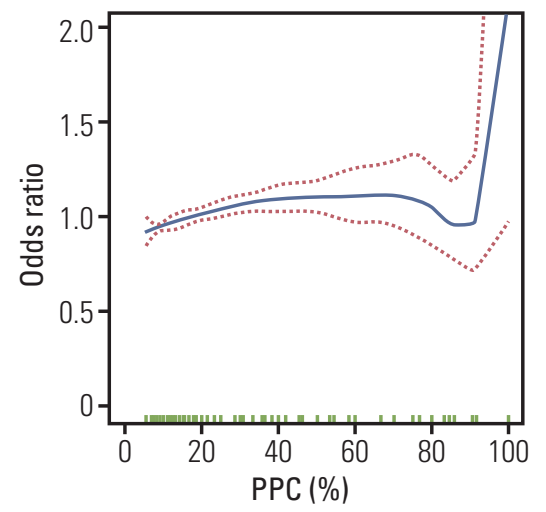

E

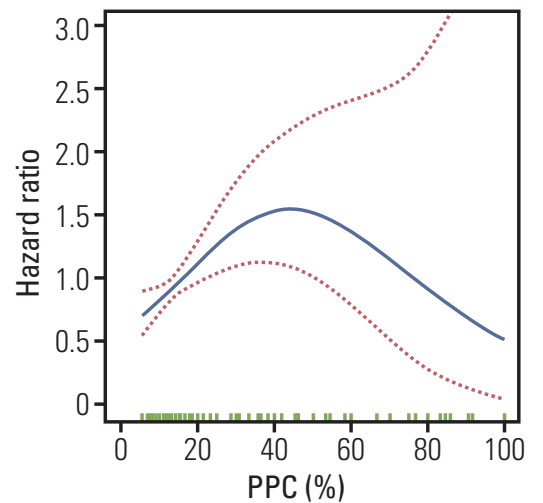

C

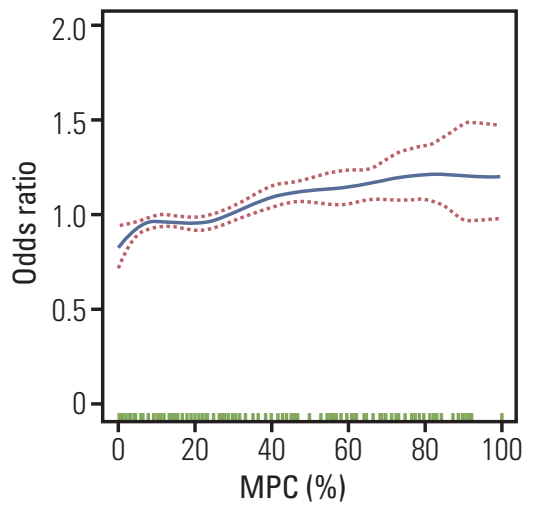

$\mathbf{F}$

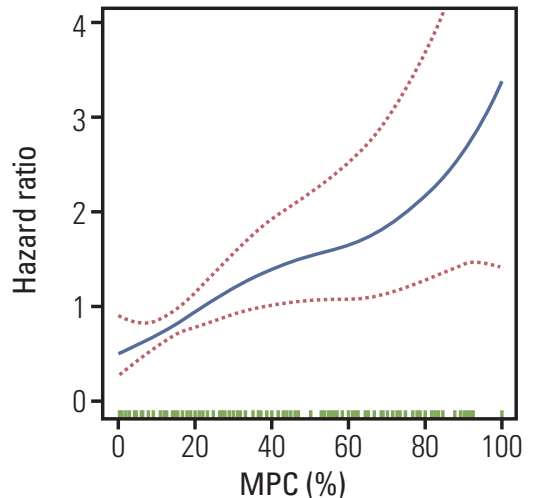

Fig. 2. Curve fitting analysis for the odds ratio for an unfavorable disease outcome (A-C) or the hazard ratio for biochemical recurrence-free survival (D-F). PSAD, prostate specific antigen density; PPC, percent positive cores; MPC, maximum cancer percentage in any core.

Table 4. Summary of our new criteria and various Western criteria for active surveillance

\begin{tabular}{lcccccc} 
Protocol & $\begin{array}{c}\text { Biopsy } \\
\text { Gleason } \\
\text { score }\end{array}$ & cStage & PSA (ng/mL) & PSAD (ng/mL/mL) & $\begin{array}{c}\text { No. of } \\
\text { positive } \\
\text { cores }\end{array}$ & $\begin{array}{c}\text { Maximum cancer } \\
\text { involvement } \\
\text { rate in any core }\end{array}$ \\
JHU (15) & $\leq 6$ & T1c & - & $<0.15$ & $\leq 2$ & $\leq 50$ \\
PRIAS (13) & $\leq 6$ & T1c-T2 & $\leq 10$ & $<0.2$ & $\leq 2$ & - \\
Univ. Miami (14) & $\leq 6$ & T1-T2 & $\leq 15$ & - & $\leq 2$ & $\leq 20$ \\
UCSF (12) & $\leq 6$ & T1-T2 & $\leq 10$ & - & $<3 \%$ & $\leq 50$ \\
MSKCC (16) & $\leq 6$ & T1-T2a & $\leq 10$ & - & - & $\leq 50$ \\
Univ. Toronto (3) & $\leq 6$ & T1-T2a & $\leq 10$ & $<0.15$ & $\leq 2$ & $\leq 20$ \\
Present study & $\leq 6$ & T1-T2a & $\leq 10$ & - & $\leq 30$ \\
\hline
\end{tabular}

cStage, clinical stage; PSA, prostate specific antigen; PSAD, PSA density; JHU, Johns Hopkins University; PRIAS, Prostate Cancer Research International: Active Surveillance; Univ. Miami, University of Miami; UCSF, University of California at San Francisco; MSKCC, Memorial Sloan-Kettering Cancer Center; Univ. Toronto, University of Toronto. 
Table 5. Key pathological outcome and biochemical recurrence survival by various active surveillance eligibility criteria

\begin{tabular}{|c|c|c|c|c|c|c|c|c|}
\hline \multirow[b]{2}{*}{ Protocol } & \multicolumn{6}{|c|}{ No $(\%)$} & \multirow{2}{*}{$\begin{array}{c}\text { 5-Year } \\
\text { BCRFS } \\
(\%)\end{array}$} & \multirow{2}{*}{$\begin{array}{c}10-Y e a r \\
\text { BCRFS } \\
(\%)\end{array}$} \\
\hline & Patient & NOCD & Upgrading & $\begin{array}{l}\text { Upgrading } \\
\geq p(4+3)\end{array}$ & $\begin{array}{c}\text { Unfavorable } \\
\text { disease }\end{array}$ & $\begin{array}{l}\text { Insignificant } \\
\text { cancer }\end{array}$ & & \\
\hline JHU (15) & $381(23.5)$ & $25(6.6)$ & $151(39.6)$ & $26(6.8)$ & $51(13.4)$ & $33(8.7)$ & 97.2 & 74.5 \\
\hline PRIAS (13) & 858 (52.9) & $82(9.6)$ & $363(42.3)$ & $69(8.0)$ & $136(15.9)$ & $74(8.7)$ & 94.2 & 83.0 \\
\hline Univ. Miami (14) & 707 (43.6) & $49(6.9)$ & $300(42.4)$ & $55(7.8)$ & $94(13.3)$ & $64(9.2)$ & 94.5 & 90.6 \\
\hline UCSF (12) & $1,127(69.4)$ & $120(10.6)$ & $531(47.1)$ & $95(8.4)$ & 197 (17.5) & $78(7.0)$ & 93.3 & 81.0 \\
\hline MSKCC (16) & $1,113(68.6)$ & $119(10.7)$ & $523(47.0)$ & $95(8.5)$ & 196 (17.6) & $78(7.1)$ & 93.7 & 79.6 \\
\hline Univ. Toronto (3) & $1,623(100)$ & $228(14.0)$ & $813(50.1)$ & $147(9.1)$ & $336(20.7)$ & $95(6.0)$ & 91.9 & 79.9 \\
\hline $\begin{array}{l}\text { Our new criteria } \\
\text { (training sample) }\end{array}$ & $402(24.8)$ & $26(6.5)$ & $140(34.8)$ & $27(6.7)$ & 47 (11.7) & $40(10.1)$ & 94.6 & 91.7 \\
\hline $\begin{array}{l}\text { Our new criteria } \\
\text { (validation sample) }\end{array}$ & 135 (26.8) & $5(3.7)$ & $50(37.0)$ & $3(2.2)$ & $8(5.9)$ & $16(12.0)$ & 98.3 & 96.7 \\
\hline
\end{tabular}

NOCD, non-organ confined disease; BCRFS, biochemical recurrence-free survival, Johns Hopkins University; JHU, Johns Hopkins University; PRIAS, Prostate Cancer Research International: Active Surveillance; Univ. Miami, University of Miami; UCSF, University of California at San Francisco; MSKCC, Memorial Sloan-Kettering Cancer Center; Univ. Toronto, University of Toronto.

ria was not based on concrete evidence, and were instead defined from existing criteria. In contrast, we defined our AS selection criteria based on research evidence and clinical rationale derived from a large scale, multicenter dataset.

Application of our findings is limited by the retrospective study design. Additionally, lack of central pathological review as well as the 2005 revision of the Gleason grading system, which likely influenced our results, are another limitation [28]. However, the pathologists in the participating centers were well experienced uropathologists; therefore, we believe that inter-rater variability would not be sufficient to alter our results. The heterogeneity of practice pattern for these patients across the eight included institutions could be another potential limitation. However, dedicated uro-oncologists managed these patients following standard practices in each institution; thus, this minor heterogeneity may enable generalization of the study in real practice scenario. We also confirmed minimal effects of institutions by sensitivity analysis. Recent studies have focused on the use of multi-parametric magnetic resonance imaging and the identification of new biomarkers to seek more appropriate AS selection criteria $[29,30]$. As these emerging criteria have yet to be standardized, we could not incorporate this information into our analysis. Despite these limitations, our study offers several strengths, with the participation of centers with the highest volume of assessment and treatment of PC in Korea being a definite advantage that favors the general interpretation and clinical application of our criteria. Furthermore, as our new AS criteria uses only general clinicopathological factors, it is readily applicable to clinical practice.
In conclusion, Korean men with PC who are eligible for AS according to Western criteria are at a higher risk for upgrading of the Gleason disease score, unfavorable disease outcomes and long-term BCR than Western patients with PC. Our new selection criteria could help improve AS protocols in Korean men, and possibly other Asian populations. These criteria are: a clinical T1c-2a stage; a biopsy Gleason score $\leq 6$; a pre-biopsy PSA $\leq 10 \mathrm{ng} / \mathrm{mL}$ and PSA density $<0.15$ $\mathrm{ng} / \mathrm{mL} / \mathrm{mL}$; number of positive cores $\leq 2$ among 10 or more total biopsy cores; and a maximum cancer involvement rate in any core $\leq 20 \%$. With these new selection criteria, we identified more potential candidates for AS than when using the Johns Hopkins University AS criteria, while maintaining a lower rate of unfavorable disease and higher BCR-free survival then when using the John Hopkins criteria.

Prospective studies using these criteria are now warranted in Korea. However, further studies are still needed to determine the optimal follow-up protocol and better criteria for initiation of active treatment during surveillance. Additionally, the contribution of multi-parametric prostate magnetic resonance imaging to refining the criteria will need to be evaluated and considered.

\section{Electronic Supplementary Material}

Supplementary materials are available at Cancer Research and Treatment website (http:// www.e-crt.org). 


\section{Conflicts of Interest}

Conflict of interest relevant to this article was not reported.

\section{Author Details}

${ }^{1}$ Department of Urology, Seoul National University Hospital, Seoul National University College of Medicine, Seoul, ${ }^{2}$ Department of Urology, Seoul National University Bundang Hospital, Seoul National University College of Medicine, Seongnam, ${ }^{3}$ Department of Urology, Samsung Medical Center, Sungkyunkwan University
School of Medicine, Seoul, ${ }^{4}$ Department of Urology, Asan Medical Center, University of Ulsan College of Medicine, Seoul, ${ }^{5}$ Department of Urology, Chonnam National University Hwasun Hospital, Chonnam National University Medical School, Gwangju, ${ }^{6}$ Department of Urology, Pusan National University Hospital, Pusan National University School of Medicine, Busan, ${ }^{7}$ Department of Urology, Kyungpook National University School of Medicine, Daegu, ${ }^{8}$ Department of Urology, Inje University Haeundae Paik Hospital, Busan, ${ }^{9}$ Department of Urology, Chonbuk National University Medical School, Jeonju, Korea

\section{References}

1. Heidenreich A, Bastian PJ, Bellmunt J, Bolla M, Joniau S, van der Kwast T, et al. EAU guidelines on prostate cancer. Part 1: screening, diagnosis, and local treatment with curative intentupdate 2013. Eur Urol. 2014;65:124-37.

2. Mohler JL, Kantoff PW, Armstrong AJ, Bahnson RR, Cohen M, D'Amico AV, et al. Prostate cancer, version 2.2014. J Natl Compr Canc Netw. 2014;12:686-718

3. Klotz L, Vesprini D, Sethukavalan P, Jethava V, Zhang L, Jain $\mathrm{S}$, et al. Long-term follow-up of a large active surveillance cohort of patients with prostate cancer. J Clin Oncol. 2015;33: 272-7.

4. D'Amico AV, Whittington R, Malkowicz SB, Schultz D, Blank $\mathrm{K}$, Broderick GA, et al. Biochemical outcome after radical prostatectomy, external beam radiation therapy, or interstitial radiation therapy for clinically localized prostate cancer. JAMA. 1998;280:969-74.

5. Byun SS, Lee S, Lee SE, Lee E, Seo SI, Lee HM, et al. Recent changes in the clinicopathologic features of Korean men with prostate cancer: a comparison with Western populations. Yonsei Med J. 2012;53:543-9.

6. Jeong CW, Jeong SJ, Hong SK, Lee SB, Ku JH, Byun SS, et al. Nomograms to predict the pathological stage of clinically localized prostate cancer in Korean men: comparison with western predictive tools using decision curve analysis. Int J Urol. 2012;19:846-52.

7. Kang M, Jeong CW, Choi WS, Park YH, Cho SY, Lee S, et al. Pre- and post-operative nomograms to predict recurrence-free probability in korean men with clinically localized prostate cancer. PLoS One. 2014;9:e100053.

8. Kim TH, Jeon HG, Choo SH, Jeong BC, Seo SI, Jeon SS, et al Pathological upgrading and upstaging of patients eligible for active surveillance according to currently used protocols. Int J Urol. 2014;21:377-81.

9. Lee DH, Jung HB, Lee SH, Rha KH, Choi YD, Hong SJ, et al. Comparison of pathological outcomes of active surveillance candidates who underwent radical prostatectomy using contemporary protocols at a high-volume Korean center. Jpn J Clin Oncol. 2012;42:1079-85.
10. Chung MS, Lee SH. Current status of active surveillance in prostate cancer. Investig Clin Urol. 2016;57:14-20.

11. Ploussard G, Epstein JI, Montironi R, Carroll PR, Wirth M, Grimm MO, et al. The contemporary concept of significant versus insignificant prostate cancer. Eur Urol. 2011;60:291-303.

12. Jeong SJ, Yeon JS, Lee JK, Cha WH, Jeong JW, Lee BK, et al. Development and validation of nomograms to predict the recovery of urinary continence after radical prostatectomy: comparisons between immediate, early, and late continence. World J Urol. 2014;32:437-44.

13. Whitson JM, Porten SP, Hilton JF, Cowan JE, Perez N, Cooperberg MR, et al. The relationship between prostate specific antigen change and biopsy progression in patients on active surveillance for prostate cancer. J Urol. 2011;185:1656-60.

14. van den Bergh RC, Roemeling S, Roobol MJ, Roobol W, Schroder FH, Bangma CH. Prospective validation of active surveillance in prostate cancer: the PRIAS study. Eur Urol. 2007;52:1560-3.

15. Soloway MS, Soloway CT, Eldefrawy A, Acosta K, Kava B, Manoharan M. Careful selection and close monitoring of lowrisk prostate cancer patients on active surveillance minimizes the need for treatment. Eur Urol. 2010;58:831-5.

16. Carter HB, Kettermann A, Warlick C, Metter EJ, Landis P, Walsh PC, et al. Expectant management of prostate cancer with curative intent: an update of the Johns Hopkins experience. J Urol. 2007;178:2359-64.

17. Adamy A, Yee DS, Matsushita K, Maschino A, Cronin A, Vickers $\mathrm{A}$, et al. Role of prostate specific antigen and immediate confirmatory biopsy in predicting progression during active surveillance for low risk prostate cancer. J Urol. 2011;185: 477-82.

18. Torre LA, Bray F, Siegel RL, Ferlay J, Lortet-Tieulent J, Jemal A. Global cancer statistics, 2012. CA Cancer J Clin. 2015;65: 87-108.

19. Jeong IG, Dajani D, Verghese M, Hwang J, Cho YM, Hong JH, et al. Differences in the aggressiveness of prostate cancer among Korean, Caucasian, and African American men: a retrospective cohort study of radical prostatectomy. Urol Oncol. 
2016;34:3.

20. Raymundo EM, Rice KR, Chen Y, Zhao J, Brassell SA. Prostate cancer in Asian Americans: incidence, management and outcomes in an equal access healthcare system. BJU Int. 2011;107: 1216-22.

21. Reese AC, Landis P, Han M, Epstein JI, Carter HB. Expanded criteria to identify men eligible for active surveillance of low risk prostate cancer at Johns Hopkins: a preliminary analysis. J Urol. 2013;190:2033-8.

22. Mizuno K, Inoue T, Kinoshita H, Yano T, Kawanishi H, Kanda $\mathrm{H}$, et al. Evaluation of predictors of unfavorable pathological features in men eligible for active surveillance using radical prostatectomy specimens: a multi-institutional study. Jpn J Clin Oncol. 2016;46:1156-61.

23. Bokhorst LP, Valdagni R, Rannikko A, Kakehi Y, Pickles T, Bangma $\mathrm{CH}$, et al. A decade of active surveillance in the PRIAS study: an update and evaluation of the criteria used to recommend a switch to active treatment. Eur Urol. 2016;70:954-60.

24. Sugimoto M, Hirama H, Yamaguchi A, Koga H, Hashine K, Ninomiya I, et al. Should inclusion criteria for active surveillance for low-risk prostate cancer be more stringent? From an interim analysis of PRIAS-JAPAN. World J Urol. 2015;33: 981-7.
25. Choo SH, Jeon HG, Jeong BC, Seo SI, Jeon SS, Choi HY, et al. Predictive factors of unfavorable prostate cancer in patients who underwent prostatectomy but eligible for active surveillance. Prostate Int. 2014;2:70-5.

26. Lim SK, Kim KH, Shin TY, Chung BH, Hong SJ, Choi YD, et al. Yonsei criteria: a new protocol for active surveillance in the era of robotic and local ablative surgeries. Clin Genitourin Cancer. 2013;11:501-7.

27. Kakehi Y, Kamoto T, Shiraishi T, Ogawa O, Suzukamo Y, Fukuhara S, et al. Prospective evaluation of selection criteria for active surveillance in Japanese patients with stage T1cN0M0 prostate cancer. Jpn J Clin Oncol. 2008;38:122-8.

28. Epstein JI, Allsbrook WC Jr, Amin MB, Egevad LL; ISUP Grading Committee. The 2005 International Society of Urological Pathology (ISUP) Consensus Conference on Gleason Grading of Prostatic Carcinoma. Am J Surg Pathol. 2005;29:1228-42.

29. Jeong CW, Park YH, Hwang SI, Lee S, Jeong SJ, Hong SK, et al. The role of 3-tesla diffusion-weighted magnetic resonance imaging in selecting prostate cancer patients for active surveillance. Prostate Int. 2014;2:169-75.

30. Park BH, Jeon HG, Choo SH, Jeong BC, Seo SI, Jeon SS, et al. Role of multiparametric 3.0-Tesla magnetic resonance imaging in patients with prostate cancer eligible for active surveillance. BJU Int. 2014;113:864-70. 